\section{Revista de CIENCIAS AMBIENTALES Tropical Journal of Environmental Sciences}

Revista de Ciencias Ambientales (Trop J Environ Sci). EISSN: 2215-3896. (Enero-Junio, 2019) . Vol 53(1): 70-91. Dol: http://dx.doi.org/10.15359/rca.53-1.4 Open Access: www.revistas.una.ac.cr/ambientales EMAlL: revista.ambientales@una.ac.cr

Barquero-Elizondo A. I., Aguilar-Monge I., Méndez-Cartín A.L,, Hernández-Sánchez G., Sánchez-Toruño H., Montero-Flores W., Herrera-González E., Sánchez-Chaves L. A., Barrantes-Vásquez A., Gutiérrez-Leitón M., Mesén-Montano I., Bullé-Bueno F.

\title{
Asociación entre abejas sin aguijón (Apidae, Meliponini) y la flora del bosque seco en la región norte de Guanacaste, Costa Rica
}

\author{
Association Between Stingless Bees (Apidae, Meliponini) and the Dry Forest Flora \\ in the Northern Region of Guanacaste, Costa Rica
}

\begin{abstract}
Ana Isabel Barquero-Elizondo ${ }^{1}$, Ingrid Aguilar-Monge ${ }^{2}$ Ana Lucía Méndez-Cartín', Gustavo Hernández-Sánchez ${ }^{4}$, Henry Sánchez-Toruño ${ }^{5}$, William Montero-Flores ${ }^{6}$, Eduardo Herrera-González ${ }^{7}$ Luis Alejandro Sánchez-Chaves ${ }^{8}$, Alejandra BarrantesVásquez $^{9}$, Milena Gutiérrez-Leitón ${ }^{10}$, Isaac Mesén-Montano ${ }^{11}$, Francisco Bullé-Bueno ${ }^{12}$
\end{abstract}

[Recibido: 8 de agosto, 2018. Aceptado: 16 de octubre, 2018. Corregido: 05 de noviembre, 2018. Publicado: 1 de enero, 2019]

Nota: Resultados preliminares de este artículo fueron presentados en el I Congreso Centroamericano de Ciencias de la Tierra y el Mar, organizado por la Facultad de Ciencias de la Tierra y el Mar de la Universidad Nacional. 13-15 noviembre 2017, San José, Costa Rica.

\section{Resumen}

Las abejas sin aguijón son importantes polinizadores en regiones tropicales y subtropicales; están asociadas a la flora que les proporciona alimento, sitios para anidamiento, resinas, savia y exudados. Se buscó determinar la asociación entre abejas y flora en el sector cerro El Hacha (bosque primario intervenido) y sector Pocosol (bosque secundario) del Parque Nacional Guanacaste, donde se inventariaron los nidos en 33 parcelas de $20 \times 20 \mathrm{~m}^{2}$. Se colectó polen de plantas dentro de las parcelas y alrededor de los nidos (radio de $500 \mathrm{~m}$ ) y se recolectaron y analizaron cargas corbiculares de polen de abejas atrapadas con redes entomológicas en la entrada de los nidos. En el cerro

1 Investigadora y docente del Instituto de Investigación y Servicios Forestales (INISEFOR), Universidad Nacional (UNA), Costa Rica. ORCID: 0000-0001-9670-128X, ana.barquero.elizondo@una.cr

2 Investigadora, docente y extensionista del Centro de Investigaciones Apícolas Tropicales (CINAT), Universidad Nacional (UNA), Costa Rica, ingrid.aguilar.monge@una.cr

3 Investigadora del Instituto de Investigación y Servicios Forestales (INISEFOR), Universidad Nacional (UNA), Costa Rica, analumeca89@ gmail.com

4 Investigador, docente y director del Instituto de Investigación y Servicios Forestales (INISEFOR), Universidad Nacional (UNA), Costa Rica, gustavo.hernandez.sanchez@una.cr

5 Investigador y docente del Instituto de Investigación y Servicios Forestales (INISEFOR), Universidad Nacional (UNA), Costa Rica, henry. sanchez.toruno@una.cr

6 Estudiante maestría Universidad de Federal do Pará, Brasil, montero.william@gmail.com

7 Investigador docente y extensionista del Centro de Investigaciones Apícolas Tropicales, Universidad Nacional (CINAT-UNA), Costa Rica, apieduardo@gmail.com

8 Investigador y docente del Centro de Investigaciones Apícolas Tropicales (CINAT), Universidad Nacional (UNA), Costa Rica, luis.sanchez. chaves@una.cr

9 Estudiante de licenciatura de la Escuela de Ciencias Ambientales, Universidad Nacional (UNA), Costa Rica, abav24@gmail.com

10 Funcionaria del Área de Conservación Guanacaste, Ministerio de Ambiente y Energía (SINAC-MINAE), Costa Rica, mgutierrez@acguanacaste.ac.cr

11 Estudiante de licenciatura de la Escuela de Ciencias Ambientales, Universidad Nacional (UNA), Costa Rica, isaac-mesen@hotmail.com

12 Estudiante posgrado Universidad Nacional Autónoma de México (UNAM), México, fgarciabulle@gmail.com

(c)




\section{Revista de CIENCIAS AMBIENTALES Tropical Journal of Environmental Sciences}

Revista de Ciencias Ambientales (Trop J Environ Sci). EISSN: 2215-3896. (Enero-Junio, 2019) . Vol 53(1): 70-91. D0l: http://dx.doi.org/10.15359/rca.53-1.4 Open Access: www.revistas.una.ac.cr/ambientales EMAIL: revista.ambientales@una.ac.cr

Barquero-Elizondo A. I., Aguilar-Monge L., Méndez-Cartín A.L., Hernández-Sánchez G., Sánchez-Toruño H., Montero-Flores W., Herrera-González E., Sánchez-Chaves L. A., Barrantes-Vásquez A., Gutiérrez-Leitón M., Mesén-Montano I., Bullé-Bueno F.

El Hacha se colectó polen de tres nidos de Scaptotrigona pectoralis y en Pocosol se colectó polen de dos nidos de Trigona fulviventris. Se obtuvo una densidad de 4.43 nidos $/ \mathrm{ha}^{-1}$, de las especies: Tetragonisca angustula, Tetragona ziegleri, Plebeia frontalis y Trigona fulviventris. Los nidos se encuentran en los troncos de árboles, con preferencia por el Quercus oleoides, donde se halló el $50 \%$ de estos. Las plantas alimenticias utilizadas por S. pectoralis en el cerro El Hacha, fueron: Cupania guatemalensis (30 \%), Byrsonima crassifolia (21\%) y Cedrela odorata (15\%). Mientras para T. fulviventris en el sector Pocosol las plantas más importantes fueron Baltimora recta (26\%), Dorstenia contrajerva (9\%) y Desmodium sp. (9\%). Es evidente el importante papel que cumple la especie arbórea Quercus oleoides para la conservación de las poblaciones de estos insectos.

Palabras clave: Abejas nativas; bosque tropical; polinización.

\begin{abstract}
Stingless bees are important pollinators in tropical and subtropical regions; they are associated with the flora that provides them with food, sites for nesting, resins, sap and exudates. We sought to determine the association between bees and flora in the cerro El Hacha sector (intervened primary forest) and the Pocosol sector (secondary forest) of the Guanacaste National Park, where the nests were inventoried in 33 plots of 20 x $20 \mathrm{~m}^{2}$. Pollen from plants was collected within the plots and around the nests (radius $500 \mathrm{~m}$ ); also, we collected and analyzed corbicular charges of pollen from bees trapped with entomological nets at the entrance of the nests. In the cerro El Hacha sector, pollen was collected from three nests of Scaptotrigona pectoralis and in the Pocosol sector we collected pollen from two nests of Trigona fulviventris. A density of 4.43 nests / ha- 1 was obtained from the species: Tetragonisca angustula, Tetragona ziegleri, Plebeia frontalis and Trigona fulviventris. The nests were found in the trunks of trees, with preference for Quercus oleoides, where $50 \%$ of them were found. The food plants used by S. pectoralis in cerro El Hacha were: Cupania guatemalensis (30 \%), Byrsonima crassifolia (21\%) and Cedrela odorata (15\%). While for T. fulviventris in the Pocosol sector the most important plants were Baltimora recta (26\%), Dorstenia contrajerva (9 $\%)$ and Desmodium sp. (9\%). The important role played by the tree species Quercus oleoides for the conservation of the populations of these insects is evident.
\end{abstract}

Keywords: Native bees; pollination; tropical forest.

\title{
1. Introducción
}

Las abejas sin aguijón (ASA) se encuentran en las zonas tropicales y subtropicales del mundo, principalmente en Sur América, donde se halla la mayor diversidad de especies (Prendas, 2013). En total se conocen cerca de 400 especies (Freitas et al., 2009), de las cuales 300 se encuentran en el Neotrópico, desde México hasta Argentina; en Costa Rica se han identificado al menos 20 géneros y 59 especies, lo cual representa cerca del $15 \%$ de la diversidad mundial de estos insectos (Aguilar, 2013; Figueroa-Mata et al., 2016).

Las ASA juegan un importante papel en la polinización de las plantas y en la agricultura en particular (Araújo et al., 2004; Batista et al., 2016, Frankie et al., 2018); su labor polinizadora contribuye a la formación de frutos y semillas y, por tanto, a la provisión de alimento para la fauna frugívora, tanto silvestre como doméstica e incluso para poblaciones humanas (Sparagnino

(cc)




\section{Revista de CIENCIAS AMBIENTALES Tropical Journal of Environmental Sciences}

Revista de Ciencias Ambientales (Trop J Environ Sci). EISSN: 2215-3896. (Enero-Junio, 2019) . Vol 53(1): 70-91. DOl: http://dx.doi.org/10.15359/rca.53-1.4 Open Access: www.revistas.una.ac.cr/ambientales EMAIL: revista.ambientales@una.ac.cr

Barquero-Elizondo A. I., Aguilar-Monge L., Méndez-Cartín A.L., Hernández-Sánchez G., Sánchez-Toruño H., Montero-Flores W., Herrera-González E., Sánchez-Chaves L. A., Barrantes-Vásquez A., Gutiérrez-Leitón M., Mesén-Montano I., Bullé-Bueno F.

et al., 2014). Son, además, una importante alternativa al uso de las abejas Apis mellifera en la polinización comercial de cultivos agrícolas (Slaa et al., 2006).

Se desconocen las razones por las cuales las abejas sin aguijón seleccionan un determinado sitio para establecer sus nidos, o la densidad de colonias que pueden desarrollarse de manera adecuada en un espacio determinado. Varios estudios de esos tópicos concluyen que, en el caso de los insectos sociales que con frecuencia viven en colonias permanentes y muy pobladas, la densidad de colonias locales puede verse limitada por la disponibilidad de sitios adecuados para anidar (Eltz et al., 2002); a cambio, los árboles, reciben el beneficio de la polinización (Armbruster, 1984, citado por Roubik, 2006). Wille (1983) menciona que la mayoría de los árboles de las selvas tropicales no son polinizados por el viento, sino por otros agentes, entre ellos las abejas que juegan un papel importante; él pudo comprobar la presencia de ASA polinizando árboles del dosel en Corcovado, Costa Rica e indica que, sin ellas, las comunidades de árboles de la selva tropical serían muy modificadas.

Vergara et al. (1986) mencionan que la mayoría de las especies de ASA prefiere árboles vivos para establecer sus nidos y la mayor altura a la que encontraron un nido en su estudio fue a $12 \mathrm{~m}$ del suelo. En relación con la densidad de colonias, Ravelo et al. (2014) estudiaron en Cuba la Melipona beechii en parcelas de $400 \mathrm{~m}^{2}$ de distintas formaciones vegetales y encontraron 1.75 nidos $/ \mathrm{ha}^{-1}$ en vegetación de mogotes, 0.86 nidos $/ \mathrm{ha}^{-1}$ en pinares, 1.24 nidos $/ \mathrm{ha}^{-1}$ en bosque semideciduo, 0.71 nidos $/$ ha $^{-1}$ en encinares y 0.45 nidos $/$ ha $^{-1}$ en bosque de galería, concluyendo que la densidad puede estar asociada al tipo de formación vegetal, a la posibilidad de encontrar alimento, protección y de alcanzar valores altos de reproducción. Por su parte, Eltz et al. (2002) encontraron en los bosques de dipterocarpáceas de Malasia una densidad de nidos alta (media de 8.4 nidos $/ \mathrm{ha}^{-1}$ ) en los sectores que bordean manglares y densidades bajas (entre 0 y 2.1 nidos/ha ${ }^{-1}$, promedio de 0.5 nidos $/ \mathrm{ha}^{-1}$ ) en los bosques continuos; estos datos muestran que la densidad de nidos es muy variable entre diferentes formaciones vegetales y hábitats en un mismo ecosistema.

Con respecto a las especies de árboles que las ASA utilizan para establecer sus nidos, Roubik (1983) menciona que estos insectos prefieren las especies de madera dura a las de madera suave, pero se desconoce si las abejas seleccionan las cavidades de los árboles según las especies. Ravelo et al., (2014) mencionan que las abejas sin aguijón usan un número de especies arbóreas relativamente bajo para la nidificación; Van Veen (1999) analizó 19 colonias de Melipona beecheii en Costa Rica, las cuales anidaban en siete diferentes especies de árboles, con una preferencia marcada por Gliricidia sepium (madero negro), lo cual coincide con los hallazgos de Moreno y Cardozo (1997), quienes concluyeron que, entre las especies arbóreas de importancia para $M$. beecheii en el establecimiento de sus nidos, se encuentran Gliricidia sepium y Bursera simaruba. Fonte et al. (2009) también determinaron una aparente preferencia de la $M$. beecheii por anidar en troncos de Gliricidia sepium, razón por la cual resaltan el importante papel de esa leguminosa forrajera utilizada en cercas vivas, en el mantenimiento de la biodiversidad en los sistemas de producción.

\begin{tabular}{|c|c|c|}
\hline 可称 & (c) (i) (5) () & 72 \\
\hline
\end{tabular}




\section{Revista de CIENCIAS AMBIENTALES Tropical Journal of Environmental Sciences}

Revista de Ciencias Ambientales (Trop J Environ Sci). EISSN: 2215-3896. (Enero-Junio, 2019) . Vol 53(1): 70-91. D0l: http://dx.doi.org/10.15359/rca.53-1.4 Open Access: www.revistas.una.ac.cr/ambientales EMAIL: revista.ambientales@una.ac.cr

Barquero-Elizondo A. I., Aguilar-Monge L., Méndez-Cartín A.L., Hernández-Sánchez G., Sánchez-Toruño H., Montero-Flores W., Herrera-González E., Sánchez-Chaves L. A., Barrantes-Vásquez A., Gutiérrez-Leitón M., Mesén-Montano I., Bullé-Bueno F.

Varias investigaciones han estudiado las abejas nativas en ecosistemas de bosque seco en Guanacaste; por ejemplo, Frankie et al. (1997) responsabilizan a la destrucción del hábitat y el desarrollo agrícola como los principales factores causantes de la disminución de la riqueza y abundancia de especies de abejas nativas en sus sitios de estudio, situación que evidenciaron incluso en remanentes boscosos de áreas protegidas. Mencionan que la presencia de periodos lluviosos con baja precipitación, seguidos de períodos de sequías, en combinación con el cambio de uso del suelo, son responsables de la declinación general de las poblaciones de abejas nativas en las tierras bajas de Guanacaste. Estos datos son más preocupantes, al considerar, como lo establecen Frankie et al. (2018), que en Costa Rica hay cerca de 8500 especies de plantas de floración y cerca de un $60 \%$ de ellas son polinizadas por especies de abejas nativas.

El presente estudio busca documentar las interacciones entre especies de abejas meliponidas y las plantas que utilizan como sitio de anidación y alimento, en tres diferentes ecosistemas del PNG, Costa Rica. Asimismo, se procedió a caracterizar la vegetación arbórea y arbustiva asociada a este importante grupo de insectos y a realizar el análisis palinológico de las cargas de polen portadas por las abejas o recolectadas en sus nidos.

\section{Metodología}

\subsection{Sitio de estudio}

El estudio se llevó a cabo en el Área de Conservación Guanacaste (ACG), Parque Nacional Guanacaste (PNG), en los sectores Pocosol y cerro El Hacha (Figura 1). En el sector Pocosol se trabajó en bosque secundario (BS) en diferentes estados de sucesión; se tiene referencias de que esos sectores fueron abandonados desde hace 39 y 56 años, lo cual propició su proceso de regeneración. Se ubica en las coordenadas geográficas N 10 88' 90” y W 85 58' 60", a 255 m s.n.m., en la zona de vida bosque húmedo premontano transición a basal, según la clasificación de Holdridge (Tosi, 1969). En este sitio la precipitación promedio anual es de $1800 \mathrm{~mm}$ y la temperatura media anual de $28{ }^{\circ} \mathrm{C}$ (Solano y Villalobos, s.f.); presenta un periodo seco que va de noviembre a abril (o de diciembre a mayo, dependiendo de la influencia del ENOS).

El sector cerro El Hacha presenta un bosque primario intervenido (BPI) y se ubica en las

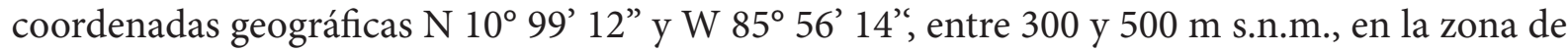
vida bosque húmedo tropical, según la clasificación de Holdridge (Tosi, 1969), con una precipitación promedio anual de $1800 \mathrm{~mm}$ y una temperatura media anual de $28{ }^{\circ} \mathrm{C}$, cuyo período seco va de noviembre a abril (Solano y Villalobos, s. f.).

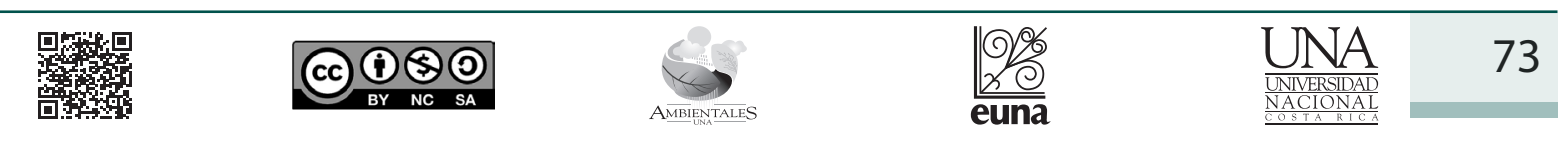




\section{Revista de CIENCIAS AMBIENTALES Tropical Journal of Environmental Sciences}

Revista de Ciencias Ambientales (Trop J Environ Sci). EISSN: 2215-3896. (Enero-Junio, 2019). Vol 53(1): 70-91. DOl: http://dx.doi.org/10.15359/rca.53-1.4 Open Access: www.revistas.una.ac.cr/ambientales EMAlL: revista.ambientales@una.ac.cr Barquero-Elizondo A. I., Aguilar-Monge I., Méndez-Cartín A.L., Hemández-Sánchez G., Sánchez-Toruño H., Montero-Flores W., Herere-Gonzzález E., Sánchez-Chaves L.A.,Barrantes-Vásquez A, Gutiérez-Leitón M., Mesén-Montanol, Bullée-Bueno F.

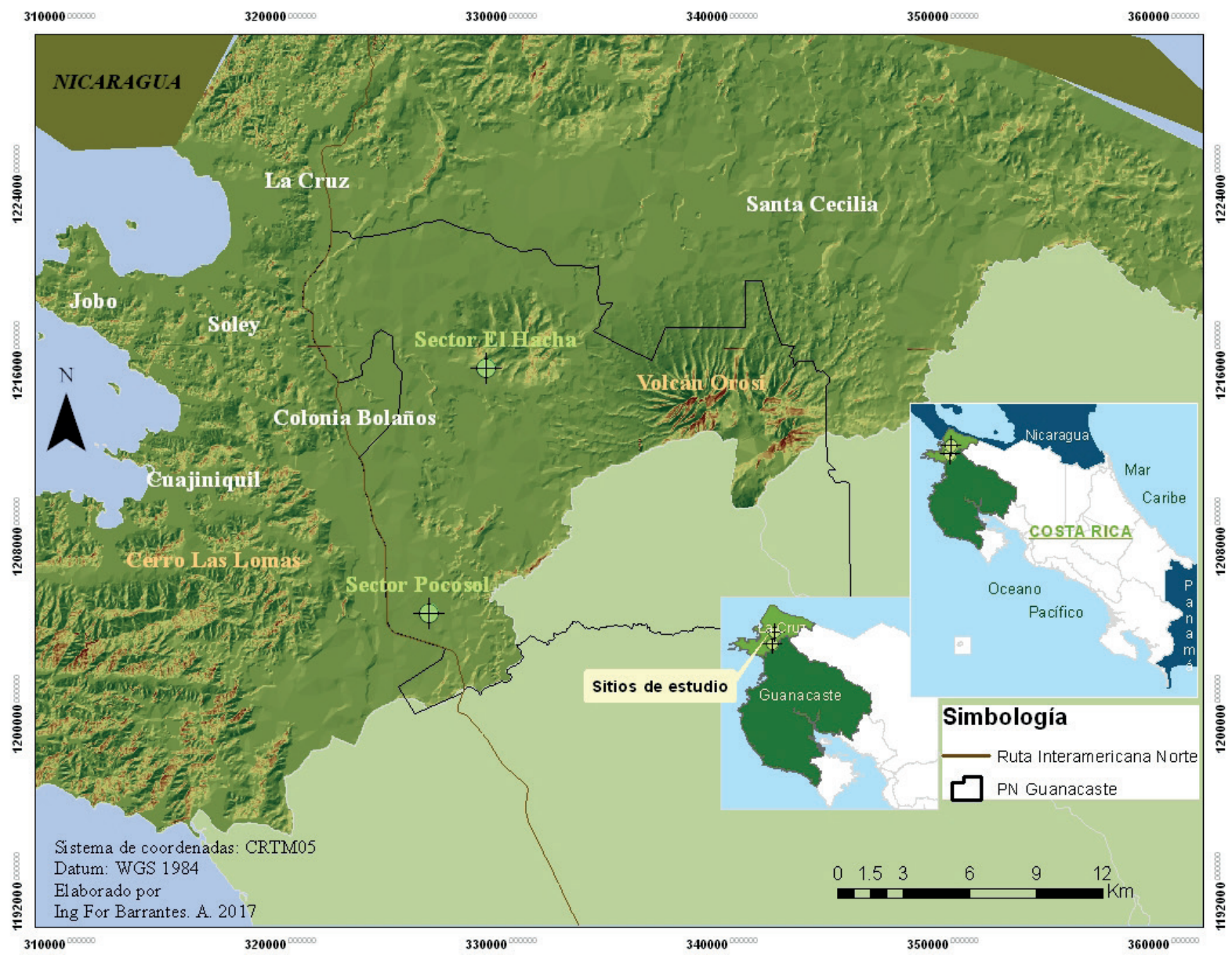

Figura 1. Ubicación del área de estudio y los sitios de muestreo (Sector Pocosol y El Hacha), Área de Conservación Guanacaste, Costa Rica.

\subsection{Disponibilidad de sitios potenciales para anidación}

En el bosque secundario del sector Pocosol la topografía es plana y los suelos son entisoles; en cada sector ( 39 y 52 años) se inventariaron once parcelas de 20 x $20 \mathrm{~m}\left(400 \mathrm{~m}^{2}\right)$ para un área total de muestreo de 0.88 ha. Por su parte, en el cerro El Hacha se muestrearon once parcelas de $400 \mathrm{~m}^{2}(0.44 \mathrm{ha}$ ) en lo que corresponde al bosque primario intervenido (Figura 2a).

Dado que se cuenta con parcelas permanentes de muestreo (PPM) en bosques secundarios de dos diferentes estados sucesionales, se buscó establecer si existen diferencias en las características del bosque y en la presencia, tanto de nidos como de especies de ASA. Por tal razón, se procedió a documentar las características dasométricas de todos los individuos, según el protocolo establecido para este fin (Sánchez, 2013).

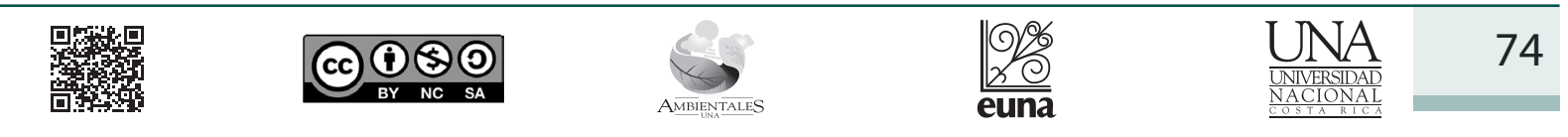




\section{Revista de CIENCIAS AMBIENTALES Tropical Journal of Environmental Sciences}

Revista de Ciencias Ambientales (Trop J Environ Sci). EISSN: 2215-3896. (Enero-Junio, 2019) . Vol 53(1): 70-91. DOl: http://dx.doi.org/10.15359/rca.53-1.4 Open Access: www.revistas.una.ac.cr/ambientales EMAIL: revista.ambientales@una.ac.cr

Barquero-Elizondo A. I., Aguilar-Monge L., Méndez-Cartín A.L., Hernández-Sánchez G., Sánchez-Toruño H., Montero-Flores W., Herrera-González E., Sánchez-Chaves L. A., Barrantes-Vásquez A., Gutiérrez-Leitón M., Mesén-Montano I., Bullé-Bueno F.

En cada parcela se midió el diámetro a $1.3 \mathrm{~m}$ de altura (DAP) de todos los individuos con dap $\geq 10 \mathrm{~cm}$ (árboles); se registró si se encontraba vivo o muerto en pie y se verificó la información taxonómica, colectando muestras botánicas en caso de que fuera necesario corroborar la taxonomía (Figura 2b). Se remidieron todos los árboles que ya estaban marcados y mapeados, y se incluyeron los nuevos ingresos, o sea, aquellos árboles que para esta medición ya habían alcanzado los $10 \mathrm{~cm}$ de DAP.

\subsection{Densidad y distribución espacial de nidos, abejas y colecta de polen}

Para cada árbol censado con la metodología descrita en la sección anterior, se determinó, visualmente, la presencia de nidos. Cada nido se georreferenció por medio de un receptor GPS marca Garmin, modelo GPSmap 60CSx, y se registró información como la especie de ASA, altura sobre el tronco a la cual se encontraba el nido, tipo y altura de la piquera (entrada del nido) (Figura 2c).

Mediante la utilización de redes entomológicas se colectaron abejas en la entrada de los nidos, las cuales fueron colocadas en pequeños tubos (viales) y trasladadas al laboratorio para su identificación taxonómica, mediante la utilización de claves y comparación con especímenes de la colección de referencia de abejas del CINAT.

Para la determinación de las plantas alimenticias utilizadas por las abejas, se realizó un muestreo en tres nidos de la especie Scaptotrigona pectoralis ubicados en los alrededores de las PPM del cerro El Hacha; lo anterior para tener más representatividad en el muestreo, al estar ubicados los nidos de esta especie cercanos entre sí y accesibles en un mismo sector. El muestreo se realizó en la época seca, en tres visitas al área de estudio durante los meses de febrero, marzo y abril de 2015. Durante cada visita se recolectaron 20 abejas obreras por nido. La mitad en un muestreo matutino de las 8:00 a las 11:00 h y la otra mitad en uno vespertino de 15:00 a 18:00 h. Las abejas fueron colectadas durante su llegada al nido, para lo cual se verificó que tuvieran polen en las corbículas. Un total de 180 muestras fueron colectadas (Figura 2d).

En la época lluviosa se muestrearon abejas obreras pecoreadoras en el sector Pocosol, en dos nidos de la especie Trigona fulviventris ubicados igualmente cercanos entre sí y accesibles para verificar el muestreo durante el mismo periodo de tiempo. Estos se muestrearon durante los meses de setiembre y octubre del año 2015, coincidiendo en este caso con el periodo de lluvias. Se colectó un total de 30 abejas por nido al día (tres días en setiembre y dos en octubre), con una distribución aproximada de la mitad de las abejas colectadas entre las 7:00 y las 10:00 h y el resto de las 14:00 a las 17:00 h, ya que su actividad de pecoreo tiende a disminuir en las horas en que el sol se encuentra en su punto más alto, debido a la elevación de la temperatura (Aguilar y Smith, 2009).

Adicionalmente, en cada una de las visitas, coincidiendo con los periodos de muestreo de abejas, se colectó el polen de plantas en flor dentro de las PPM, para lo que se utilizó, como área de muestreo, transectos aleatorios alrededor de los nidos, tomando, como referencia promedio, un radio óptimo de $500 \mathrm{~m}$, acorde con la actividad de pecoreo de las abejas. Este polen, a su vez,

\begin{tabular}{|c|c|c|}
\hline 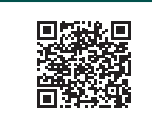 & (c) (i) (5) (2) & 75 \\
\hline
\end{tabular}




\section{Revista de CIENCIAS AMBIENTALES Tropical Journal of Environmental Sciences}

Revista de Ciencias Ambientales (Trop J Environ Sci). EISSN: 2215-3896. (Enero-Junio, 2019) . Vol 53(1): 70-91. DOl: http://dx.doi.org/10.15359/rca.53-1.4 Open Access: www.revistas.una.ac.cr/ambientales EMAlL: revista.ambientales@una.ac.cr

Barquero-Elizondo A. I., Aguilar-Monge l., Méndez-Cartín A.L., Hernández-Sánchez G., Sánchez-Toruño H., Montero-Flores W., Herrera-González E., Sánchez-Chaves L. A., Barrantes-Vásquez A., Gutiérrez-Leitón M., Mesén-Montano I., Bullé-Bueno F.

sirvió como material de referencia para su comparación con el polen que se clasificó a partir de las cargas portadas por las abejas.
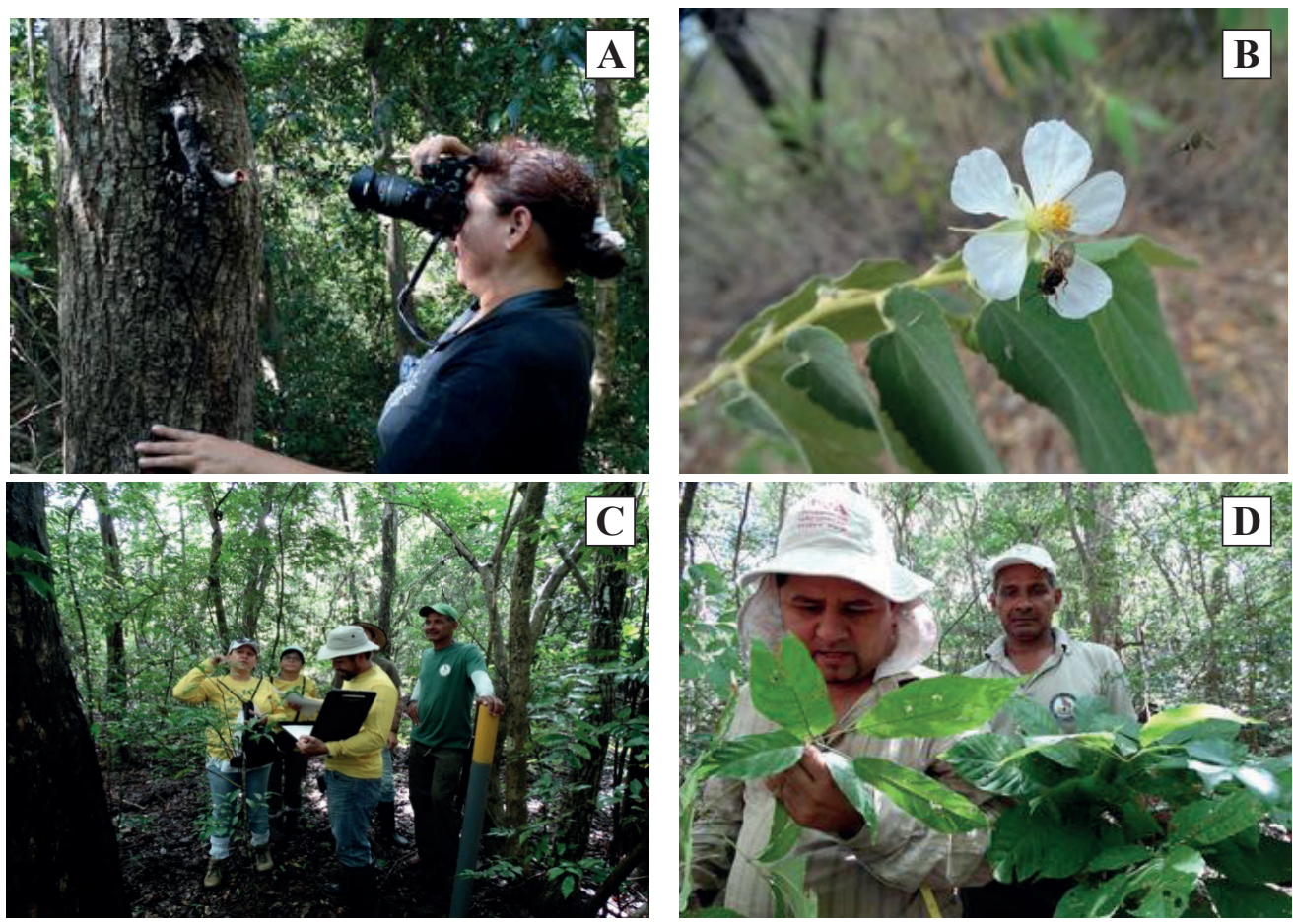

Figura 2. a) I. Aguilar fotografiando nido de Scaptotrigona pectoralis en el sector Pocosol; b) Pecoreo de S. pectoralis sobre flor de Muntingia calibura; c) Parte del equipo en parcela de bosque secundario, sector Pocosol; d) W. Montero y funcionario SINAC en determinación taxonómica

\subsection{Análisis palinológico}

Para determinar las especies de flora de las cuales las abejas portaban su polen, se utilizaron métodos de análisis palinológico que permitieron establecer, mediante las características morfológicas del grano de polen (polaridad, tamaño, aberturas en la exina y tipo de ornamentación), la ubicación taxonómica a nivel de familia, género y especie. Dichos resultados fueron verificados mediante la comparación de las microfotografías de los granos con las claves taxonómicas de granos de polen del neotrópico, así como la colección de referencia de granos de polen de las plantas del sitio y la del CINAT. Los granos de polen fueron procesados mediante la utilización del método de tinción con safranina (Sánchez, 2001). Este método utiliza las cargas de polen colectadas por las abejas en sus corbículas. Asimismo, en el caso del muestreo de T. fulviventris, se modificó dicho método, optando por embeber a la abeja por completo en una solución de jalea de glicerina, previamente teñida con safranina para extraer cualquier grano de polen adherido a su cuerpo; la solución fue colocada en láminas fijas de vidrio y homogenizada mediante calor,

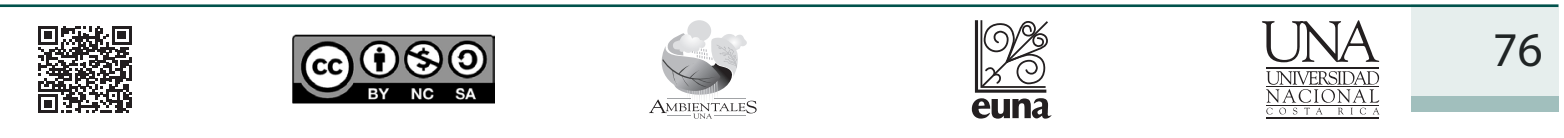




\section{Revista de CIENCIAS AMBIENTALES Tropical Journal of Environmental Sciences}

Revista de Ciencias Ambientales (Trop J Environ Sci). EISSN: 2215-3896. (Enero-Junio, 2019) . Vol 53(1): 70-91. D0l: http://dx.doi.org/10.15359/rca.53-1.4 Open Access: www.revistas.una.ac.cr/ambientales EMAIL: revista.ambientales@una.ac.cr

Barquero-Elizondo A. I., Aguilar-Monge L., Méndez-Cartín A.L., Hernández-Sánchez G., Sánchez-Toruño H., Montero-Flores W., Herrera-González E., Sánchez-Chaves L. A., Barrantes-Vásquez A., Gutiérrez-Leitón M., Mesén-Montano I., Bullé-Bueno F.

para su posterior observación e identificación del polen al microscopio. Esta modificación se realizó debido a que durante la época de muestreo no se observaron cargas de polen corbiculares en esta especie de abeja.

Para el análisis del polen colectado de las plantas en flor, se realizó una disección y separación de las anteras y estructuras florales de cada una de las especies colectadas e identificadas con anterioridad; las anteras se colocaron en cajas petri rotuladas para evitar la combinación del polen de cada planta, se introdujeron en un beaker con $15 \mathrm{ml}$ de agua destilada para llevar a ebullición a una temperatura de $400{ }^{\circ} \mathrm{C}$, con el propósito de separar los granos de polen de las anteras. Posteriormente se realizó una filtración de la solución para separar las estructuras florales de mayor tamaño, la solución filtrada se vertió en tubos de ensayo utilizando una balanza para igualar volúmenes antes de centrifugar durante siete minutos a $3500 \mathrm{rpm}$, para luego eliminar el líquido sobrenadante por decantación de un golpe; este paso se repitió dos veces para recuperar la mayor parte del polen por sedimentación. Adicionalmente, se preparó una solución de glicerol (50\% glicerina $+50 \%$ agua, $25 \mathrm{ml}$ respectivamente), la cual permite que el polen se mantenga fresco durante más tiempo, se adicionaron 10 gotas de safranina a cada tubo, para teñir las células vivas y se colocaron los tubos en un espacio carente de luz, para lograr que la solución de safranina penetrara en la pared de la célula del polen. De igual forma los tubos se centrifugaron, decantaron y dejaron en reposo en posición completamente vertical; de esta manera, los granos de polen quedan embebidos en la parte superior del tubo. Posteriormente se colocaron a secar en la estufa a $60^{\circ} \mathrm{C}$ durante 15 minutos. Finalmente, con la ayuda de una aguja de disección, se frotó con porciones pequeñas de gel de glicerina en la parte superior del tubo, donde se encontraban concentrados los granos de polen y se procedió a elaborar las láminas fijas para la observación del polen al microscopio.

\section{Resultados}

Con respecto a la composición florística y área basal de los sitios de estudio, se determinaron las características de cada tipo de bosque, según se indica en el Cuadro 1. Los datos sobre composición florística de los tres sitios en su conjunto indican la presencia de 37 familias, 70 géneros y 84 especies de árboles con DAP $\geq 10 \mathrm{~cm}$, donde es evidente la riqueza de la familia Fabaceae en los tres sitios de estudio. La cantidad de especies y la riqueza por familias fue muy similar en los dos tipos de bosque secundario, a pesar de existir una diferencia de 17 años entre uno y otro. En el Apéndice 1 se muestra la lista con los nombres comunes y científicos de todas las especies arbóreas inventariadas en las tres parcelas.

\begin{tabular}{|c|c|c|}
\hline 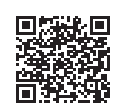 & (cc) (i) (\$) () & 77 \\
\hline
\end{tabular}




\section{Revista de CIENCIAS AMBIENTALES Tropical Journal of Environmental Sciences}

Revista de Ciencias Ambientales (Trop J Environ Sci). EISSN: 2215-3896. (Enero-Junio, 2019) . Vol 53(1): 70-91. D0l: http://dx.doi.org/10.15359/rca.53-1.4 Open Access: www.revistas.una.ac.cr/ambientales EMAIL: revista.ambientales@una.ac.cr

Barquero-Elizondo A. I., Aguilar-Monge I., Méndez-Cartín A.L,, Hernández-Sánchez G., Sánchez-Toruño H., Montero-Flores W., Herrera-González E., Sánchez-Chaves L. A., Barrantes-Vásquez A., Gutiérrez-Leitón M., Mesén-Montano I., Bullé-Bueno F.

Cuadro 1. Características del bosque y composición florística por sitio de estudio

\begin{tabular}{|c|c|c|c|c|c|c|}
\hline Sector & $\begin{array}{l}\text { Tipo de } \\
\text { bosque }\end{array}$ & $\begin{array}{c}\text { N.o } \\
\text { indiv ha } \\
\end{array}$ & $\begin{array}{c}\text { Área basal } \\
\mathbf{m}^{2} \mathbf{h a}^{-1}\end{array}$ & $\begin{array}{l}\text { N. }{ }^{\circ} \text { familias } \\
\text { en } 0.44 \text { ha }\end{array}$ & $\begin{array}{c}\text { N.o de } \\
\text { especies }\end{array}$ & $\begin{array}{c}\text { Familias más ricas } \\
\text { (número de especies) }\end{array}$ \\
\hline Pocosol 1 & $\begin{array}{l}\text { Secundario } \\
\text { de } 39 \text { años }\end{array}$ & 491 & 30.4 & 22 & 40 & $\begin{array}{l}\text { Fabaceae/Papilionoideae (5), } \\
\text { Rubiaceae (5) y Salicaceae (4) }\end{array}$ \\
\hline \multirow{3}{*}{ Pocosol 2} & \multirow{3}{*}{$\begin{array}{l}\text { Secundario } \\
\text { de } 56 \text { años }\end{array}$} & \multirow{3}{*}{495} & \multirow{3}{*}{27.6} & \multirow{3}{*}{22} & \multirow{3}{*}{41} & Fabaceae/Papilionoideae (5) \\
\hline & & & & & & Rubiaceae (5) \\
\hline & & & & & & Anacardiaceae (4) \\
\hline Cerro El Hacha & $\begin{array}{l}\text { Primario } \\
\text { intervenido }\end{array}$ & 498 & 32.3 & 31 & 57 & $\begin{array}{l}\text { Fabaceae/Papilionoideae (6), } \\
\text { Rubiaceae (5) y Boraginaceae (4) } \\
\text { Malvaceae (4) }\end{array}$ \\
\hline
\end{tabular}

Adicionalmente, en los alrededores de las parcelas, se realizaron colectas de plantas en floración, lo cual dio como resultado la presencia de 30 especies adicionales, entre ellas una especie arbórea, nueve arbustos, doce hierbas, cinco lianas herbáceas y tres lianas leñosas. La lista completa de las especies mencionadas se muestra en el Apéndice 2.

El bosque secundario de 56 años muestra una mayor cantidad de individuos por ha con respecto al bosque secundario de 35 años, pero una disminución en el área basal por ha; el bosque primario intervenido es el que presenta mayor cantidad de individuos y mayor área basal por ha. Por otra parte, en el bosque secundario de Pocosol la especie Q. oleoides es la que presenta el mayor índice de valor de importancia (IVI $=69.71$ ), lo cual significa el $23.23 \%$ del total del IVI e indica la importancia ecológica relativa de la especie en este ecosistema. La situación es similar en el cerro El Hacha, pues la especie Q. oleoides alcanza un IVI de 43.10, correspondiente al 14 $\%$ del IVI del bosque primario intervenido.

Dado que los datos de composición florística y área basal mostraron pocas diferencias en los tres sitios de estudio, se optó por analizar los datos del inventario de nidos de ASA de manera conjunta, lo cual dio como resultado que se localizaron un total de 35 nidos de abejas sin aguijón, más una colmena de Apis mellifera, para una densidad total de 4.43 nidos/ ha ${ }^{-1}$ (Figura 3).

En el bosque primario intervenido se hallaron las especies Scaptotrigona pectoralis, Partamona orizabaensis, Plebeia frontalis y Plebeia sp., las cuales fueron exclusivas de ese tipo de ecosistema; solamente una especie de ASA se ubicó en los tres tipos de bosque estudiados: Trigona fulviventris. En el bosque secundario (sectores 1 y 2 de Pocosol) se logró ubicar las especies Tetragonisca angustula, Tetragona ziegleri y Nannotrigona perilampoides (Figura 3).

(cc)




\section{Revista de CIENCIAS AMBIENTALES Tropical Journal of Environmental Sciences}

Revista de Ciencias Ambientales (Trop J Environ Sci). EISSN: 2215-3896. (Enero-Junio, 2019) . Vol 53(1): 70-91. D0l: http://dx.doi.org/10.15359/rca.53-1.4 Open Access: www.revistas.una.ac.cr/ambientales EMAIL: revista.ambientales@una.ac.cr Barquero-Elizondo A. I., Aguilar-Monge I., Méndez-Cartín A.L,, Hernández-Sánchez G., Sánchez-Toruño H., Montero-Flores W., Herrera-González E., Sánchez-Chaves L. A., Barrantes-Vásquez A., Gutiérrez-Leitón M., Mesén-Montano I., Bullé-Bueno F.

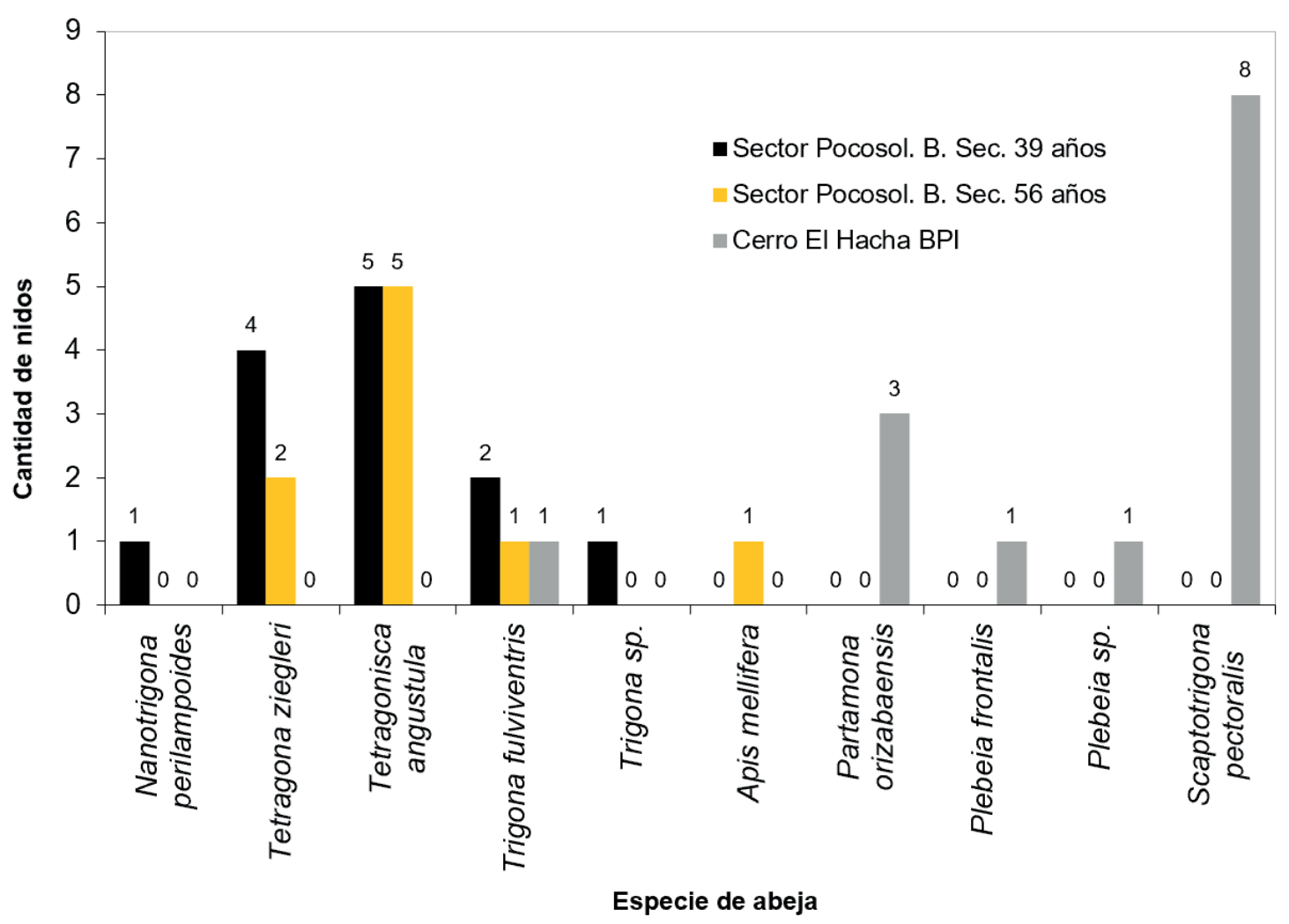

Figura 3. Cantidad de nidos por especie de abeja en cada sector.

En la Figura 4 se muestra la preferencia de las abejas por las distintas especies arbóreas. El $50 \%$ de los nidos se encontraron en el Quercus oleoides, además de ello, seis de las 10 especies de abejas los construyeron en los troncos de esta misma especie. Todos los nidos de Partamona orizabaensis fueron construidos en los troncos del Ficus sp. En troncos de árboles muertos se ubicaron tres nidos de tres especies de ASA (Tetragona ziegleri, Tetragonisca angustula y Trigona sp.). La especie arbórea Rehdera trinervis, un árbol de porte bajo, de fuste acanalado, que permite a las ASA construir sus nidos en medio de los intersticios del tronco, fue la segunda especie más utilizada, con un $14 \%$ de los nidos. Las abejas de la especie Tetragonisca angustula utilizaron varias especies arbóreas para construir sus nidos (cinco los hicieron en el encino y uno en cada una de las siguientes especies: Bursera simaruba, Lonchocarpus rugosus, Tabebuia ochraceae, Rehedera trinervis y en un árbol de especie indeterminada, pues se encontraba seco.

Las especies de abejas con mayor cantidad de nidos (considerando los tres sitios) fueron: Tetragonisca angustula $(\mathrm{n}=10)$; Scaptotrigona pectoralis $(\mathrm{n}=8)$; Tetragona ziegleri $(\mathrm{n}=6)$; Trigona fulviventris $(\mathrm{n}=3)$ y Partamona orizabaensis $(\mathrm{n}=3)$.

\begin{tabular}{|c|c|c|}
\hline 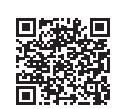 & (c) (i) (5) (O) & 79 \\
\hline
\end{tabular}




\section{Revista de CIENCIAS AMBIENTALES Tropical Journal of Environmental Sciences}

Revista de Ciencias Ambientales (Trop J Environ Sci). EISSN: 2215-3896. (Enero-Junio, 2019) . Vol 53(1): 70-91. DOl: http://dx.doi.org/10.15359/rca.53-1.4 Open Access: www.revistas.una.ac.cr/ambientales EMAIL: revista.ambientales@una.ac.cr

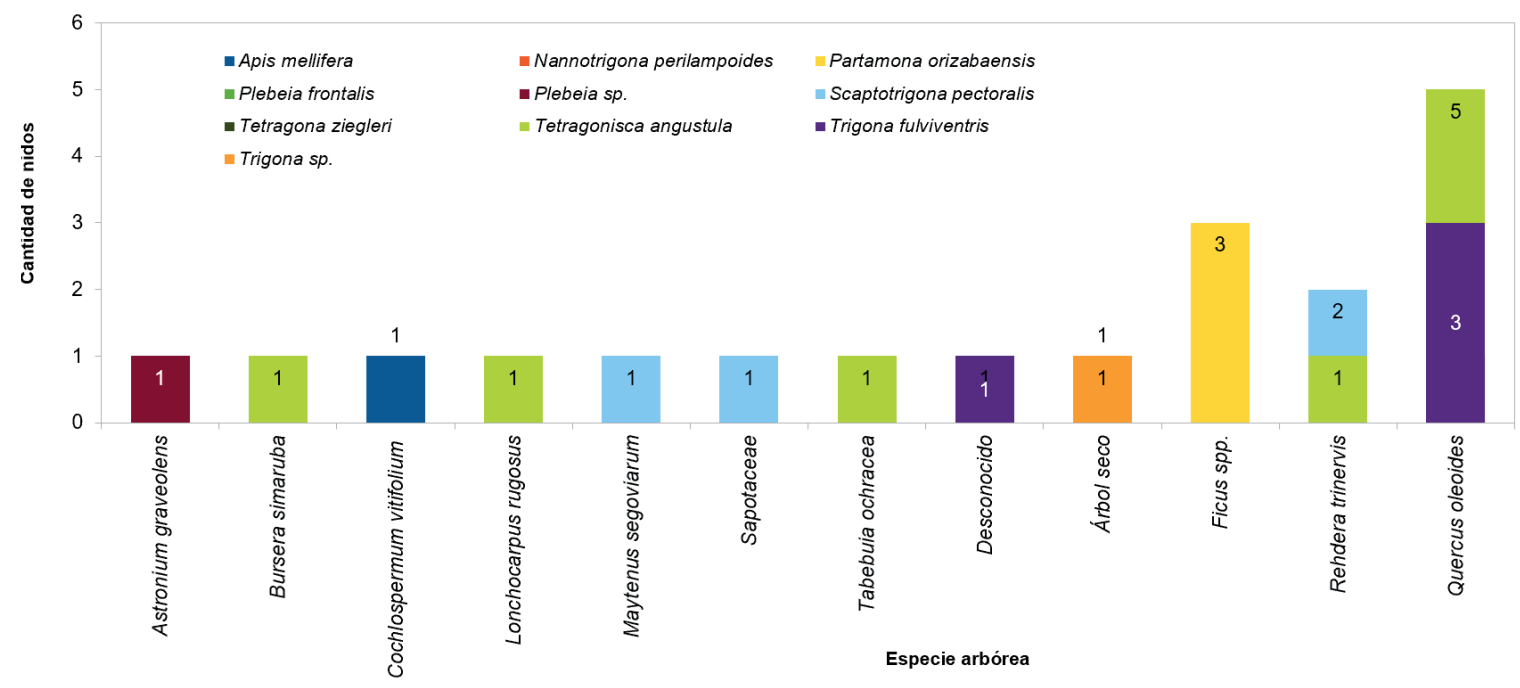

Figura 4. Cantidad de nidos por especie de abeja y por especie arbórea.

La Figura 5 muestra la selección del sitio (por especie de abeja) según el DAP del árbol donde construyeron sus nidos. La especie Partamona orizabaensis fue la que utilizó el árbol más grueso, de más de $2 \mathrm{~m}$ de DAP (la especie arbórea no se indica en el gráfico, pero se trató de un Ficus sp.); por su parte la especie Scaptotrigona pectoralis utilizó diferentes estratos del bosque para ubicar sus nidos, pues estos mismos se encontraron desde alturas menores a un metro hasta los $12 \mathrm{~m}$ de altura, resultado coincidente con lo reportado por Vergara et al. (1986). La especie T. fulviventris seleccionó alturas a nivel del suelo (tierra o raíces); los nidos de Tetragonisca angustula que se logró inventariar estaban ubicados desde el nivel del suelo hasta alturas menores a 3.5 metros. 


\section{Revista de

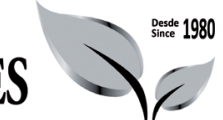 Tropical Journal of Environmental Sciences}

Revista de Ciencias Ambientales (Trop J Environ Sci). EISSN: 2215-3896. (Enero-Junio, 2019) . Vol 53(1): 70-91. D0l: http://dx.doi.org/10.15359/rca.53-1.4 Open Access: www.revistas.una.ac.cr/ambientales EMAIL: revista.ambientales@una.ac.cr Barquero-Elizondo A. I., Aguilar-Monge L., Méndez-Cartín A.L., Hernández-Sánchez G., Sánchez-Toruño H., Montero-Flores W., Herrera-González E., Sánchez-Chaves L. A., Barrantes-Vásquez A., Gutiérrez-Leitón M., Mesén-Montano I., Bullé-Bueno F.

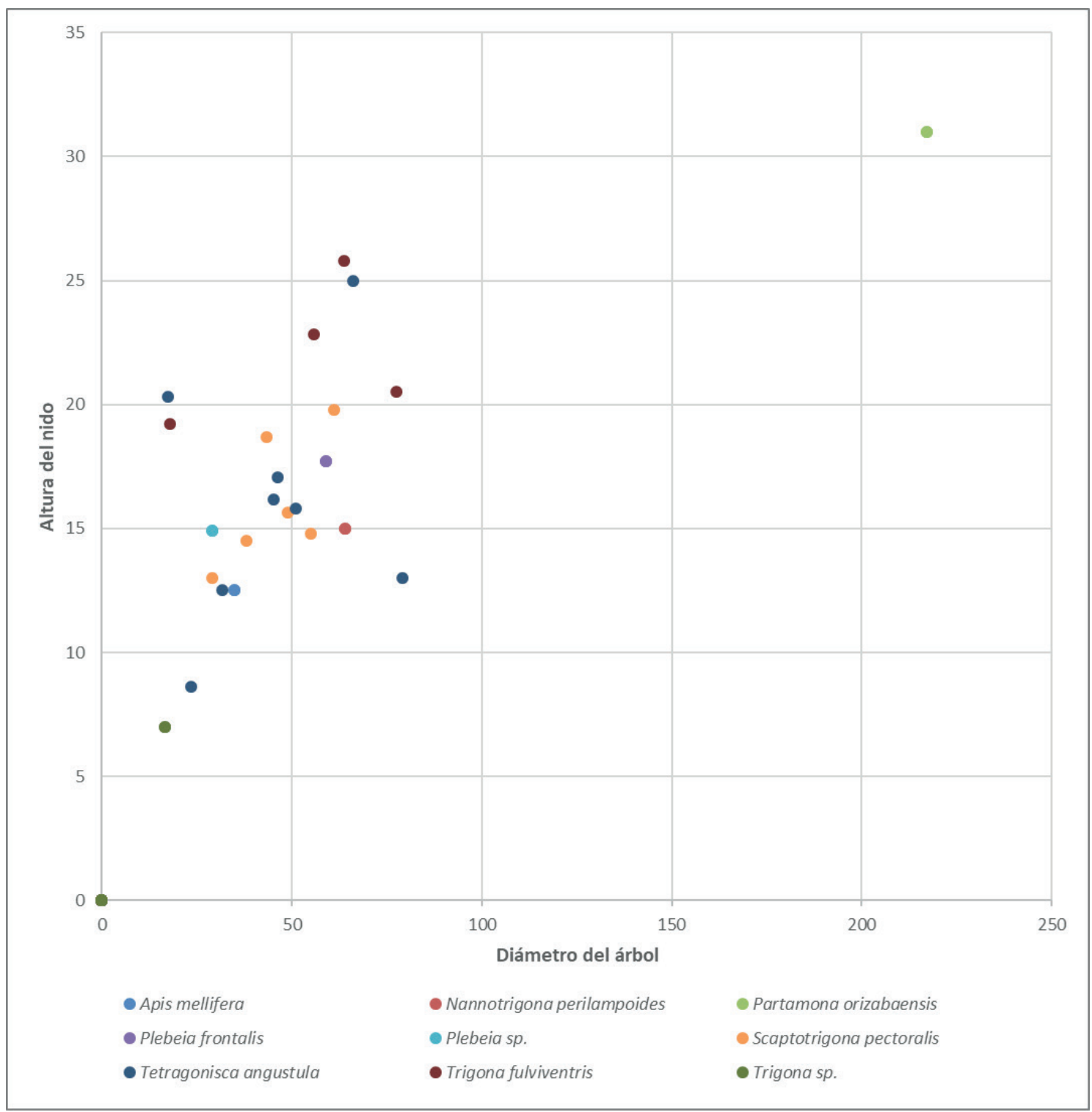

Figura 5. Altura de los nidos por especie de ASA y diámetro de los árboles donde los construyeron.

En el estudio palinológico realizado con muestras de polen de Scaptotrigona pectoralis del cerro El Hacha y Trigona fulviventris del sector Pocosol, de las 184 muestras analizadas, 32 de ellas (17 \%) no evidenciaron ningún tipo polínico presente. En los dos nidos de Trigona fulviventris se determinó un total de 26 tipos polínicos distintos, pertenecientes a 19 familias botánicas, de los cuales 14 fueron determinados a nivel de especie y los restantes 12 solamente a nivel
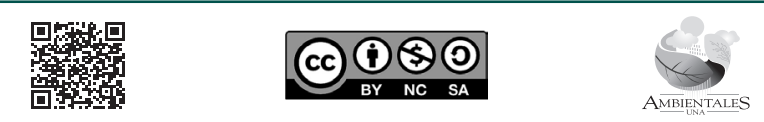


\section{Revista de CIENCIAS AMBIENTALES Tropical Journal of Environmental Sciences}

Revista de Ciencias Ambientales (Trop J Environ Sci). EISSN: 2215-3896. (Enero-Junio, 2019) . Vol 53(1): 70-91. DOl: http://dx.doi.org/10.15359/rca.53-1.4 Open Access: www.revistas.una.ac.cr/ambientales EMAlL: revista.ambientales@una.ac.cr

Barquero-Elizondo A. I.,Aguilar-Monge I., Méndez-Cartín A.L, Hermánder-Sánchez G., Sánchez-Toruño H., Montero-Flores W., Herere-Gonzzález E., Sánchez-Chaves L.A.,Barrantes-Vásquez A, Gutiérez-Leitón M., Mesén-Montanol, Bullée-Bueno F.

de género. La familia que presentó mayor número de especies fue Fabaceae, subfamilia Papilionoideae, con cuatro especies, el resto de las familias visitadas por T. fulviventris estuvo representado en su mayoría por una o dos especies. La especie más representativa en los estudios de las cargas polínicas corresponde a Baltimora recta, una planta de pequeño porte, perteneciente a la familia Asteraceae, con una abundancia del $35 \%$ para el nido 2 y cerca del $20 \%$ para el nido 1. Los restantes recursos polínicos se encontraron en proporciones menores al $20 \%$. Entre los árboles identificados mediante el polen analizado se encuentran Astronium graveolens (ron-ron), Cecropia sp. (guarumo), Trema micrantha (jucó), Inga sp. (guaba), Andira inermis (almendro de montaña), Lonchocarpus sp. (chaperno), Miconia argentea (María colorada), Eugenia sp. y Triplaris sp. (hormigo). De esas especies solamente cuatro fueron inventariadas dentro de las parcelas (A. graveolens, M. argentea, Lonchocarpus sp. y Eugenia sp.).

El estudio del comportamiento de la especie Scaptotrigona pectoralis, en la época seca (meses de febrero, marzo y abril), mediante la colecta de abejas en tres nidos del BPI del cerro El Hacha permitió identificar un total de 26 morfotipos de polen en las 180 muestras recolectadas; de los 26 morfotipos de polen se logró identificar 17 a nivel de especie, 9 permanecieron a nivel de género de un total de 15 familias. Se determinó que las especies de árboles más visitados por Scaptotrigona pectoralis durante los muestreos fueron Cupania guatemalensis (cantarillo), Byrsonima crassifolia (nance), Eugenia sp., Miconia argentea, Brosimun alicastrum (ojoche) y en menos proporción Cedrela odorata (cedro) y Quercus oleoides (encino). Con respecto a la cantidad de polen encontrado por especie dentro de las diferentes muestras, se obtuvo que la mayor parte del polen en las muestras pertenece a las especies Byrsonima crassifolia (nance), Cupania guatemalensis (cantarillo) y Brosimun alicastrum (ojoche).

\section{Discusión}

$\mathrm{Al}$ analizar la composición florística de los tres sitios, se destaca la riqueza de la familia Fabaceae, lo cual es particularmente importante para la alimentación de las abejas, pues sus especies (que van desde plantas de pequeño porte, lianas, arbustos y árboles) presentan floraciones muy abundantes y constantes a lo largo del año.

La cantidad de especies y la riqueza por familias fue muy similar en los dos tipos de bosque secundario, a pesar de existir una diferencia de 17 años entre uno y otro. Esto puede estar asociado a que en el bosque seco los ciclos de crecimiento se ven limitados por la periodicidad de lluvias; lo que puede generar que existan pocas diferencias perceptibles en periodos de tiempo menores a 50 años.

La densidad de nidos encontrada fue de 4.43 nidos/ ha $^{-1}$; por su parte Roubik (1983), en un estudio realizado en bosque húmedo y lluvioso tropical en Panamá, encontró 6.0 nidos/ha-1; mientras que Eltz et al. (2002) encontraron en los bosques de dipterocarpaceas de Malasia, densidades altas (media de 8.4 nidos $/ \mathrm{ha}^{-1}$ ) en los sectores que bordean manglares y densidades bajas (entre 0 y 2.1 nidos $/ \mathrm{ha}^{-1}$, promedio de 0.5 nidos $/ \mathrm{ha}^{-1}$ ) en los bosques continuos.

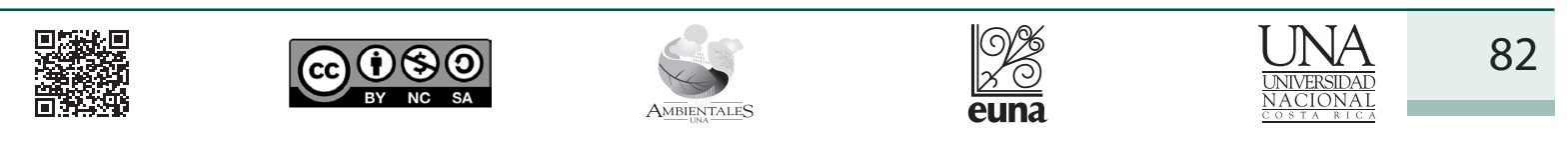




\section{Revista de CIENCIAS AMBIENTALES Tropical Journal of Environmental Sciences}

Revista de Ciencias Ambientales (Trop J Environ Sci). EISSN: 2215-3896. (Enero-Junio, 2019) . Vol 53(1): 70-91. DOl: http://dx.doi.org/10.15359/rca.53-1.4 Open Access: www.revistas.una.ac.cr/ambientales EMAIL: revista.ambientales@una.ac.cr

Barquero-Elizondo A. I., Aguilar-Monge L., Méndez-Cartín A.L., Hernández-Sánchez G., Sánchez-Toruño H., Montero-Flores W., Herrera-González E., Sánchez-Chaves L. A., Barrantes-Vásquez A., Gutiérrez-Leitón M., Mesén-Montano I., Bullé-Bueno F.

En el presente estudio, en troncos del Quercus oleoides se identificaron nidos de seis especies de ASA, lo cual da indicios de una preferencia por la especie. Estos troncos usualmente presentan cavidades, las cuales podrían originarse debido a los daños causados por los recurrentes incendios forestales de la época seca. Otras especies arbóreas también utilizadas fueron Astronium graveolens (ron-ron), Bursera simaruba (indio pelado) y Ficus sp. (higuerón). Por su parte, Roubik (1983) reportó la presencia de nidos en los troncos del Brosimum alicastrum en bosque tropical lluvioso de Panamá y Ravelo et al. (2014) determinaron que Melipona beecheii, en Cuba, hace uso de un total de 15 especies de árboles para su nidificación, entre los cuales, los más frecuentemente utilizados fueron: Bursera simaruba, Guazuma ulmifolia, Psidium guajava, Mangifera indica y la palma Roystonea regia. Leal (2010, citado por Ravelo et al., 2014) también encontró una mayor cantidad de nidos en árboles de Bursera simaruba en las formaciones boscosas galería y siempreverde en Cuba. Según lo mencionado por Aguilar et al. (2013), los árboles del género Ficus son usados por las abejas sin aguijón de Costa Rica como sustrato para el establecimiento de sus colonias.

Tal como se muestra en la Figura 3, en el BPI fue donde se encontró la mayor cantidad de nidos (14); hay cuatro especies de ASA que son exclusivas del BPI del cerro El Hacha y no se encontraron en ninguno de los bosques secundarios estudiados en el sector Pocosol. La especie Scaptotrigona pectoralis, la cual es exclusiva del BPI, fue la que presentó la mayor abundancia de nidos, con un total de ocho.

En el presente estudio, la altura de construcción de los nidos fue variable; por ejemplo, los nidos de Partamona orizabaensis se ubicaron en el dosel intermedio e inferior (4 a $7 \mathrm{~m}$ ), lo cual coincide con lo reportado por Jarau \& Barth (2008), quienes los encontraron en cavidades en el suelo, en los troncos de los árboles, entre las raíces de epífitas o incluso en nidos de aves abandonados. Llama la atención la adaptabilidad de la especie S. pectoralis, la cual utilizó diferentes estratos del bosque, desde alturas menores a un metro hasta los $12 \mathrm{~m}$ de altura; mientras que los nidos de la T. fulviventris se encontraron en el suelo o en la base de árboles, coincidiendo con lo reportado por Roubik (2006), o por Jarau \& Barth (2008) en su estudio en la región de Golfo Dulce, Costa Rica.

En el cerro El Hacha se colectaron y procesaron muestras de polen de tres nidos de Scaptotrigona pectoralis, durante el período de máxima floración (estación seca) de febrero a abril de 2015; mientras que en el sector Pocosol, las muestras de polen fueron colectadas de dos nidos de Trigona fulviventris en la época de menor floración (estación lluviosa) de setiembre a octubre de 2015.

Dado que las muestras de polen de nidos de ASA se colectaron en distintas épocas (estación seca en cerro El Hacha y estación lluviosa en sector Pocosol), esto podría estar generando diferencias muy significativas en los resultados para cada sitio, ya que la mayor explosión de floraciones, en general, se da durante la época seca (noviembre a mayo) y son pocas las especies con floración entre junio y octubre, por ejemplo, Acosmium (árbol perteneciente a la familia Fabaceae) y algunas Sapotaceas como Manilkara o Sideroxylon. Al respecto, Ramalho et al. (1990)

\begin{tabular}{|c|c|c|c|c|c|}
\hline 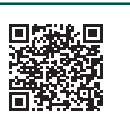 & (c) (i) (3) (2) & 8 & 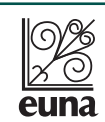 & 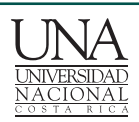 & 83 \\
\hline
\end{tabular}




\section{Revista de CIENCIAS AMBIENTALES Tropical Journal of Environmental Sciences}

Revista de Ciencias Ambientales (Trop J Environ Sci). EISSN: 2215-3896. (Enero-Junio, 2019) . Vol 53(1): 70-91. DOl: http://dx.doi.org/10.15359/rca.53-1.4 Open Access: www.revistas.una.ac.cr/ambientales EMAIL: revista.ambientales@una.ac.cr

Barquero-Elizondo A. I., Aguilar-Monge L., Méndez-Cartín A.L., Hernández-Sánchez G., Sánchez-Toruño H., Montero-Flores W., Herrera-González E., Sánchez-Chaves L. A., Barrantes-Vásquez A., Gutiérrez-Leitón M., Mesén-Montano I., Bullé-Bueno F.

indican que las preferencias en las colectas suelen estar influenciadas por la estacionalidad climática y la disposición del recurso floral en el tiempo.

Es importante indicar que los resultados obtenidos para Scaptotrigona pectoralis en el cerro El Hacha coinciden con otros estudios que revelan la importancia alimenticia de las familias Myrtaceae, Melastomataceae. Moraceae y Sapindaceae para las abejas sin aguijón en el Neotrópico (Ramalho et al., 1990; Wilms y Wiechers, 1997). A diferencia de lo reportado también en esas investigaciones, se encontraron pocas plantas de la familia Asteraceae con un papel relevante para las abejas en el sector cerro El Hacha, posiblemente debido a que se trata de un bosque en un estado más maduro, donde dicha familia está poco representada. En contraposición, en el sector Pocosol, para la especie Trigona fulviventris, la especie Baltimora recta, (familia Asteraceae) resultó ser la especie más abundante. En los diferentes muestreos realizados en Pocosol, esta planta herbácea apareció como el recurso más importante en las colectas durante la época lluviosa, lo cual brindó un indicio sobre el comportamiento fenológico de la planta, pues al presentarse un invierno más seco, influenciado por el efecto del Niño, su periodo de floración se amplió, posiblemente como repuesta adaptativa a esa variación climática. Usualmente esta planta florece en junio y julio, alrededor del "veranillo" y luego cuando las lluvias se intensifican las flores tienden a desaparecer (Espina y Ordetx, 1983).

La cantidad de familias identificadas en el estudio palinológico coincide con los resultados obtenidos por Ferreira y Absy (2013), quienes analizaron polen de Melipona (Melikerria) interrupta en el municipio de Manacapuru, Estado de Amazonas, Brasil y, en las muestras de residuos evaluadas, identificaron 32 tipos polínicos, que representaban 19 familias botánicas, y tres tipos de polen no estaban determinados. Al igual que en el presente estudio, Ferreira y Absy (2013) encontraron que la familia más representativa fue Fabaceae (Mimosoideae), con ocho tipos de polen.

\section{Conclusiones}

Desde el punto de vista florístico, la familia botánica dominante en las tres condiciones de bosque fue la Fabaceae, la cual es de gran relevancia para el ecosistema por su abundante floración, utilizada por las abejas sin aguijón como fuente de polen y néctar; aspecto que se logró comprobar, pues de los tipos polínicos identificados que portaban las abejas, cuatro pertenecían a esta familia.

La especie Quercus oleoides fue la preferida por las abejas sin aguijón como sitio de anidación, es además la que presenta el mayor IVI en todos los sectores y los mayores DAP en las PPM, por lo que cumple un papel importante para la conservación de las poblaciones de abejas sin aguijón en estos bosques.

En el caso de la Scaptotrigona pectoralis, se sugiere la existencia de una especificidad cuantitativa y cualitativa en las preferencias de visita floral de la especie. Por su parte, para Trigona fulviventris no es posible asegurar una especificidad en la dominancia presente en las colectas para

\begin{tabular}{|c|c|c|}
\hline 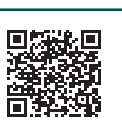 & (c) (1) () () () & 84 \\
\hline
\end{tabular}




\section{Revista de CIENCIAS AMBIENTALES Tropical Journal of Environmental Sciences}

Revista de Ciencias Ambientales (Trop J Environ Sci). EISSN: 2215-3896. (Enero-Junio, 2019) . Vol 53(1): 70-91. D0l: http://dx.doi.org/10.15359/rca.53-1.4 Open Access: www.revistas.una.ac.cr/ambientales EMAlL: revista.ambientales@una.ac.cr

Barquero-Elizondo A. I., Aguilar-Monge L., Méndez-Cartín A.L., Hernández-Sánchez G., Sánchez-Toruño H., Montero-Flores W., Herrera-González E., Sánchez-Chaves L. A., Barrantes-Vásquez A., Gutiérrez-Leitón M., Mesén-Montano I., Bullé-Bueno F.

la época del año analizada, en lo relativo a la posible relación entre esta especie y las plantas más representativas del estudio, ya que las limitaciones de recursos nutricionales que se presentaron en el sitio, en conjunto con las necesidades alimenticias de la población de las colonias, obliga a las abejas a recurrir a múltiples fuentes de nutrición.

El conocimiento generado en este estudio sobre las poblaciones de abejas sin aguijón en el ACG y su asociación con el hábitat se convierte en una herramienta para la determinación de la vulnerabilidad de estos insectos en los escenarios que se presentan, producto de la variabilidad ambiental y la acción antropogénica directa. Además, se podrán generar, en el futuro, criterios de uso sustentable y sostenible para las abejas en su entorno y favorecer, así, que continúen brindando los servicios ecosistémicos de polinización al bosque seco tropical.

\section{Agradecimientos}

El equipo autor agradece al Fondo Institucional de Desarrollo Académico (FIDA) de la Universidad Nacional (UNA) por el aporte financiero para la ejecución del proyecto "Dinámica de los ecosistemas forestales de bosque seco en Costa Rica y su impacto en las poblaciones de abejas nativas: protección de polinizadores y plantas hospederas asociadas". Al ingeniero forestal Pablo Montoya Gutiérrez, por su apoyo en el trabajo de campo, y al personal del Área de Conservación Guanacaste (sector Pocosol) y de la Estación Experimental Forestal Horizontes, por toda la colaboración brindada. Finalmente, agradecemos a la Revista y las personas revisoras anónimas por sus oportunas observaciones realizadas a la versión final del documento.

\section{Referencias}

Aguilar, I., Herrera, E. y Zamora, G. (2013). Stingless bees of Costa Rica. En Vit, P., Pedro, S. y Roubik, D. , M. Pcompletar apellido y Roubik D. Pcompletar apellido (Eds.), Pot Honey. Vit. Springer New York. Recuperado de https://link.springer.com/content/ pdf/10.1007\%2F978-1-4614-4960-7.

Aguilar Sierra, C. I. y Smith Pardo, A. H. (2009). Abejas visitantes de Mimosa pigra L. (Mimosaceae): Comportamiento de pecoreo y cargas polínicas. Acta Biológica Colombiana, 14(1), 109-120. Recuperado de http://www.scielo.org.co/pdf/abc/v14n1/v14n1a06.pdf

Araújo, E. D., Costa, M., Chaud-Netto, J. y Fowler, H. G. (2004). Body size and flight distance in stingless bees (Hymenoptera: Meliponini): inference of flight range and possible ecological implications. Brazilian Journal of Biology, 64(3B), 563-568. https://doi.org/10.1590/ S1519-69842004000400003

Batista, M., Abreu, B., Dutra, R., Cunha, M., do Amaral, F., Torres, L. y Ribeiro, M. (2016). Chemical composition and antioxidant activity of geopropolis produced by Melipona fasciculata

\begin{tabular}{|c|c|c|}
\hline 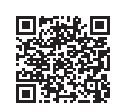 & (cc) (i) (\$) () & 85 \\
\hline
\end{tabular}




\section{Revista de CIENCIAS AMBIENTALES Tropical Journal of Environmental Sciences}

Revista de Ciencias Ambientales (Trop J Environ Sci). EISSN: 2215-3896. (Enero-Junio, 2019) . Vol 53(1): 70-91. Dol: http://dx.doi.org/10.15359/rca.53-1.4 Open Access: www.revistas.una.ac.cr/ambientales EMAIL: revista.ambientales@una.ac.cr

Barquero-Elizondo A. I.,Aguilar-Monge I., Méndez-Cartín A.L, Hermánder-Sánchez G., Sánchez-Toruño H., Montero-Flores W., Herrera-GonzálezE., Sánchez-Chaves L.A., Barrantes-Vásquez A., Gutiérez-Leitón M., Mesén-Montano I., Bullé-Bueno F.

(Meliponinae) in flooded fields and cerrado areas of Maranhão State, northeastern Brazil. Acta Amazonica, 46(3), 315-322. Recuperado de http://dx.doi.org/10.1590/1809-4392201600034

Eltz, T., Brühl, C. A. y Linsenmair, K. E. (2002). Determinants of stingless bee nest density in lowland dipterocarp forests of Sabah, Malaysia. Oecologia, 131(1), 27-34. doi: 10.1007/ s00442-001-0848-6

Espina, D. y Ordetx, G. S. (1983). Flora apícola tropical. Cartago, Costa Rica: Editorial Tecnológica de Costa Rica.

Ferreira, M. G. y Absy, M. L. (2013). Pollen analysis of the post-emergence residue of Melipona (Melikerria) interrupta Latreille (Hymenoptera: Apidae) bred in the central Amazon region. Acta Botanica Brasilica, 27(4), 709-713. https://doi.org/10.1590/S0102-33062013000400009

Figueroa-Mata, G., Prendas-Rojas, J. P., Ramírez-Bogantes, M., Aguilar-Monge, I., Herrera-González, E. y Travieso-González, C. M. (2016). Identificación de abejas sin aguijón (Apidae: Meliponini) a partir de la clasificación de los descriptores SIFT de una imagen del ala derecha anterior. Revista Tecnología en Marcha, 29(5), 51-63. https://doi.org/10.18845/tm.v29i5.2585

Fonte, L., Betancourt, D., Demedio, L. y Aguilar, I. (2009). Gliricidia sepium: Leguminosa forrajera útil como habitáculo de Melipona beecheii en sistemas agrarios. Revista Biocenosis, 22 $(1-2)$.

Frankie, G. W., Vinson, S. B., Rizzardi, M. A., Griswold, T. L., O’Keefe, S. y Snelling, R. R. (1997). Diversity and Abundance of Bees Visiting a Mass Flowering Tree Species in Disturbed Seasonal Dry Forest, Costa Rica. Journal of the Kansas Entomological Society, 70(4), 281-296.

Frankie, G. W., Vinson, S. B., Rizzardi, M. A., Griswold, T. L., Coville, R. E., Grayum, M. H., Martinez, L. E. S., Foltz-Sweat, J., y Pawelek, J. C. (2013). Relationships of Bees to Host Ornamental and Weedy Flowers in Urban Northwest Guanacaste Province, Costa Rica. Journal of the Kansas Entomological Society, 86(4), 325-351. doi: 10.2317/JKES121222.1

Frankie, G. W., Coville, R. E., Pawele, J. C., Jadallah, C. C., Vinson, S. B. y Martínez, L. E. S. (2018). Bee-Flower-People Relationships, Field Biologists, and Conservation in Northwest Urban Costa Rica and Beyond. Zoosymposia, 12, 018-028. doi: 10.11646/zoosymposia.12.1.4

Freitas, B. M., Imperatriz-Fonseca, V. L., Medina, L. M., Kleinert, A. de M. P., Galetto, L., Nates-Parra, G., y Quezada-Euán, J. J. G. (2009). Diversity, threats and conservation of native bees in the Neotropics. Apidologie, 40(3), 332-346. doi:10.1051/apido/2009012.

Jarau, S., y Barth, F. G. (2008). Stingless bees of the Golfo Dulce region, Costa Rica (Hymenoptera, Apidae, Apidae, Meliponini). Zugleich Kataloge der oberösterreichischen Landesmuseen, 80, 267-276.

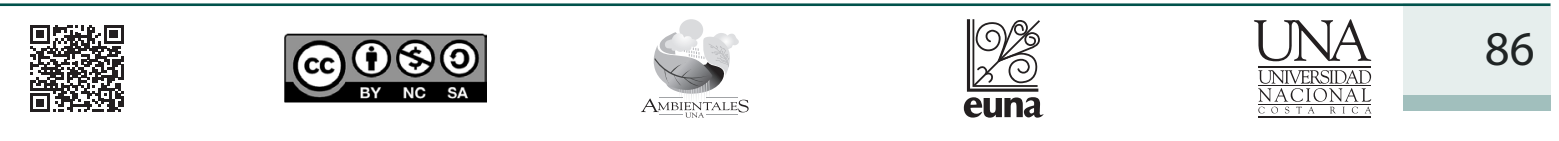




\section{Revista de CIENCIAS AMBIENTALES Tropical Journal of Environmental Sciences}

Revista de Ciencias Ambientales (Trop J Environ Sci). EISSN: 2215-3896. (Enero-Junio, 2019) . Vol 53(1): 70-91. DOl: http://dx.doi.org/10.15359/rca.53-1.4 Open Access: www.revistas.una.ac.cr/ambientales EMAIL: revista.ambientales@una.ac.cr

Barquero-Elizondo A. I., Aguilar-Monge I., Méndez-Cartín A.L., Hernández-Sánchez G., Sánchez-Toruño H., Montero-Flores W., Herrera-González E., Sánchez-Chaves L. A., Barrantes-Vásquez A., Gutiérrez-Leitón M., Mesén-Montano I., Bullé-Bueno F.

Moreno F. A. y Cardozo A. F. (1997). Abundancia de abejas sin aguijón (Meliponinae) en especies maderables del Estado de Portuguesa, Venezuela. Vida Silvestre Neotropical, 6(1-2), 53-56.

Prendas, J. P., Figueroa, G., Travieso, C., Herrera, E., Aguilar, I. y Ramírez, M. (2013). Sistema automático de clasificación de abejas sin aguijón (Apidae: Meliponini) basado en el contorno y venación de sus alas. Propuesta y avances. En Memoria del VIII Congreso Mesoamericano de abejas nativas: Biología, cultura y uso sostenible. Heredia, Costa Rica. 26 al 31 de agosto de 2013. Recuperado de http://www.cinat.una.ac.cr/VIIICongresoMesoamericano/docs/ Memoria.pdf

Ramalho, M., Kleinert-Giovannini, M. y Imperatriz-Fonseca, V. L. (1990). Important bee plants for stingless bees (Melipona and Trigonini) and Africanized honeybees (Apis mellifera) in neotropical habitats: a review. Apidologie, 21(5), 469-488. https://doi.org/10.1051/ apido:19900508

Ravelo, K., Hernández, F., Paneque, I., Toledo, L. y Gutiérrez, H. (2014). Relación de la población natural de abejas de la tierra (Melipona beecheii) con la flora en el valle San Andrés. Revista Cubana de Ciencias Forestales, 2(1), 21-34.

Roubik, D. (1983). Nest and colony characteristics stingless bees Panama. J. Kansas Ent. Soc, 56(3), 327-355.

Roubik, D. (2006). Stingless bee nesting biology. Apidologie, 37(2006), 124-143 doi: 10.1051/ apido:2006026

Sánchez, L. A. (2001). Métodos palinológicos de análisis. (Documento no publicado). Centro de Investigaciones Apícolas Tropicales (CINAT-UNA). Heredia, Costa Rica.

Sánchez, M. (2013). Protocolo de establecimiento y medición de parcelas permanentes de muestreo en bosque natural. para la Red de parcelas permanentes de monitoreo de ecosistemas forestales (RedMEF). Instituto de Investigación y Servicios Forestales. Universidad Nacional, Heredia, Costa Rica.

Slaa, E., Sánchez, L. A., Malagodi-Braga, K. y Hofstede, F. (2006). Stingless bees in applied pollination: practice and perspectives. Apidologie, 37(2006), 293-315. https://doi.org/10.1051/ apido:2006022

Solano, J. y Villalobos, R. (s. f.) Regiones y subregiones climáticas de Costa Rica. Instituto Meteorológico Nacional, San José, Costa Rica.

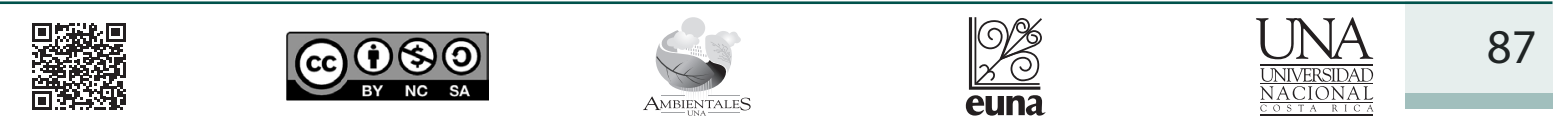




\section{Revista de CIENCIAS AMBIENTALES

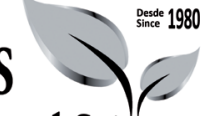 Tropical Journal of Environmental Sciences}

Revista de Ciencias Ambientales (Trop J Environ Sci). EISSN: 2215-3896. (Enero-Junio, 2019) . Vol 53(1): 70-91. Dol: http://dx.doi.org/10.15359/rca.53-1.4 Open Access: www.revistas.una.ac.cr/ambientales EMAlL: revista.ambientales@una.ac.cr Barquero-Elizondo A. I., Aguilar-Monge I., Méndez-Cartín A.L,, Hernández-Sánchez G., Sánchez-Toruño H., Montero-Flores W., Herrera-González E., Sánchez-Chaves L. A., Barrantes-Vásquez A., Gutiérrez-Leitón M., Mesén-Montano I., Bullé-Bueno F.

Sparagnino, C., Chianetta, P., y Basilio, A. M. (2014). Especies arbóreas utilizadas por abejas meliponas (Apidae: Meliponini) en el bosque chaqueño semiárido en Formosa (Argentina). Agronomía y Ambiente, 34(2014), 85-91.

Tosi, J. (1969). Mapa ecológico de Costa Rica. Instituto Geográfico Nacional, San José, Costa Rica.

Van Veen, J. W. (1999). Colony reproduction in stingless bees. Costa Rica: Litografía e imprenta Lil.

Vergara, B., Villa, A. y Nates, G. (1986). Nidificación de meliponinos (Hymenoptera: Apidae) de la Región Central de Colombia. Rev. Biol. Trop., 34(2), 181-184.

Wille, A. (1983). Biology of the stingless bees. Annual review of entomology, 28(1), 41-64. https://doi.org/10.1146/annurev.en.28.010183.000353

Wilms, W. y Wiechers, B. (1997). Floral resource partitioning between native Melipona bees and the introduced Africanized honey bee in the Brazilian Atlantic rain forest. Apidologie, 28, 339-355. https://doi.org/10.1051/apido:19970602

\section{Apéndices}

Apéndice 1. Lista de árboles inventariados en las parcelas

\begin{tabular}{|c|c|c|}
\hline Nombre científico & Familia & Nombre común \\
\hline Albizia adinocephala (Donn. Sm.) Britton y Ros & Fabaceae/Mimosoideae & Guanacaste blanco \\
\hline Amyris pinnata Kunth & Rutaceae & ND \\
\hline Annona purpurea Moc. y Sessé ex Dunal & Annonaceae & Toreta, soncoya \\
\hline Annona reticulata $\mathrm{L}$. & Annonaceae & Anona, anonillo \\
\hline Apeiba tibourbou Aubl. & Malvaceae & Peine de mico \\
\hline Ardisia revoluta Kunth & Primulaceae & Tucuico \\
\hline Astronium graveolens Jacq. & Anacardiaceae & Ron ron \\
\hline Bourreria grayumii Gottschling y J.S. Mill. & Boraginaceae & ND \\
\hline Bursera simaruba (L.) Sarg. & Burseraceae & Jiñocuabe, indio pelado \\
\hline Bursera tomentosa (Jacq.) Triana y Planch. & Burseraceae & Caraño \\
\hline Byrsonima crassifolia (L.) Kunth & Malpighiaceae & Nance \\
\hline Calycophyllum candidissimum (Vahl) DC. & Rubiaceae & Madroño \\
\hline Casearia praecox Griseb. & Salicaceae & Varasón \\
\hline Casearia sylvestris Sw. & Salicaceae & Huesillo \\
\hline Cassipourea guianensis Aubl. & Rhizophoraceae & ND \\
\hline Chomelia spinosa Jacq. & Rubiaceae & Malacahuite \\
\hline Chrysophyllum brenesii Cronquist & Chrysobalanaceae & ND \\
\hline Cochlospermum vitifolium (Willd.) Spreng. & Bixaceae & Poro-poro \\
\hline
\end{tabular}

\begin{tabular}{|c|c|}
\hline 品敉 & (c) (i) (9) () \\
\hline
\end{tabular}




\section{Revista de

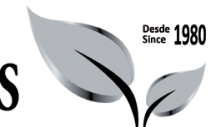 Tropical Journal of Environmental Sciences}

Revista de Ciencias Ambientales (Trop J Environ Sci). EISSN: 2215-3896. (Enero-Junio, 2019) . Vol 53(1): 70-91. D0l: http://dx.doi.org/10.15359/rca.53-1.4 Open Access: www.revistas.una.ac.cr/ambientales EMAlL: revista.ambientales@una.ac.cr

Barquero-Elizondo A. I., Aguilar-Monge I., Méndez-Cartín A.L., Hernández-Sánchez G., Sánchez-Toruño H., Montero-Flores W., Herrera-González E., Sánchez-Chaves L. A., Barrantes-Vásquez A., Gutiérrez-Leitón M., Mesén-Montano I., Bullé-Bueno F.

\begin{tabular}{|c|c|c|}
\hline Nombre científico & Familia & Nombre común \\
\hline Cordia alliodora (Ruiz y Pav.) Oken & Boraginaceae & Laurel \\
\hline Cordia bicolor A. DC. & Boraginaceae & Muñeco \\
\hline Cordia panamensis L. Riley & Boraginaceae & Muñeco \\
\hline Dalbergia retusa Hemsl. & Fabaceae/Papilionoideae & Cocobola \\
\hline Dialium guianense (Aubl.) Sandwith & Fabaceae/Caesalpinioideae & Tamarindo de montaña \\
\hline Dilodendron costaricense (Radlk.) A. H. Gentry y S & Sapindaceae & Iguano \\
\hline Erblichia odorata Seem. & Turneraceae & ND \\
\hline Eugenia hypargyrea Standl. & Myrtaceae & ND \\
\hline Exostema mexicanum A. Gray & Rubiaceae & ND \\
\hline Faramea occidentalis (L.) A. Rich. & Rubiaceae & Cafecillo \\
\hline Ficus obtusifolia Kunth & Moraceae & Higuerón \\
\hline Garcinia intermedia (Pittier) Hammel & Clusiaceae & Jorco \\
\hline Genipa americana $\mathrm{L}$. & Rubiaceae & Guaitil, tapaculo \\
\hline Gliricidia sepium (Jacq.) Kunth ex Walp. e & Fabaceae/Papilionoideae & Madero negro \\
\hline Guazuma ulmifolia Lam. & Malvaceae & Capulín \\
\hline Guettarda macrosperma Donn. Sm. & Rubiaceae & Madroño negro \\
\hline Gymnanthes lucida Sw. & Euphorbiaceae & ND \\
\hline Handroanthus chrysanthus (Jacq.) S.O. Grose & Bignoniaceae & ND \\
\hline Handroanthus impetiginosus (Mart. ex DC.) Mattos & Bignoniaceae & ND \\
\hline Handroanthus ochraceus (Cham.) Mattos & Bignoniaceae & Cortez \\
\hline Heisteria cyanocarpa Poepp. & Olacaceae & ND \\
\hline Hymenaea courbaril L. & Fabaceae/Caesalpinioideae & Guapinol \\
\hline Karwinskia calderoni Standl. & Rhamnaceae & Chiliguiste \\
\hline Krugiodendron ferreum (Vahl) Urb. & Rhamnaceae & Quiebra hacha \\
\hline Lonchocarpus acuminatus (Schltdl.) M. Sousa & Fabaceae/Papilionoideae & Chaperno \\
\hline Lonchocarpus felipei N. Zamora & Fabaceae/Papilionoideae & Chaperno \\
\hline Lonchocarpus minimiflorus Donn. Sm. & Fabaceae/Papilionoideae & Chapernillo \\
\hline Lonchocarpus rugosus Benth & Fabaceae/Papilionoideae & Carao macho \\
\hline Lonchopcarpus oliganthus F.J. Herm. & Fabaceae/Papilionoideae & Chaperno \\
\hline Luehea speciosa Willd. & Malvaceae & Guácimo \\
\hline Lysiloma divaricatum (Jacq.) J. F. Macbr. & Fabaceae/Mimosoideae & Quebracho \\
\hline Mabea montana Müll. Arg. & Euphorbiaceae & ND \\
\hline Mabea occidentalis Benth. & Euphorbiaceae & ND \\
\hline Machaerium biovulatum Micheli & Fabaceae/Papilionoideae & Espino negro \\
\hline Manilkara chicle (Pittier) Gilly & Sapotaceae & Níspero chicle \\
\hline Margaritaria nobilis L. f. & Phyllanthaceae & ND \\
\hline
\end{tabular}

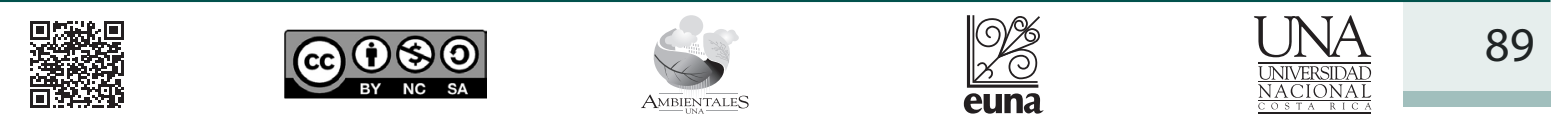




\section{Revista de CIENCIAS AMBIENTALES Tropical Journal of Environmental Sciences}

Revista de Ciencias Ambientales (Trop J Environ Sci). EISSN: 2215-3896. (Enero-Junio, 2019) . Vol 53(1): 70-91. Dol: http://dx.doi.org/10.15359/rca.53-1.4 Open Access: www.revistas.una.ac.cr/ambientales EMAIL: revista.ambientales@una.ac.cr

Barquero-Elizondo A. I., Aguilar-Monge I., Méndez-Cartín A.L., Hernández-Sánchez G.S Sánchez-Toruño H., Montero-Flores W., Herrera-González E., Sánchez-Chaves L. A., Barrantes-Vásquez A., Gutiérrez-Leitón M., Mesén-Montano I., Bullé-Bueno F.

\begin{tabular}{|c|c|c|}
\hline Nombre científico & Familia & Nombre común \\
\hline Maytenus segoviarum Standl. y L.O. Williams & Celastraceae & ND \\
\hline Miconia argentea (Sw.) DC. & Melastomataceae & María colorada \\
\hline Mouriri myrtilloides (Sw.) Poir. & Melastomataceae & Huesillo \\
\hline Nectandra salicina C.K. Allen & Lauraceae & Ira \\
\hline Ocotea veraguensis (Meisn.) Mez & Lauraceae & Canelillo, canelo \\
\hline Pilocarpus racemosus Vahl & Rutaceae & Talcacao \\
\hline Posoqueria latifolia (Rudge) Roem. y Schult. & Rubiaceae & Guayaba de mono \\
\hline Pouteria reticulata (Engl.) Eyma & Sapotaceae & Níspero zapote \\
\hline Pseudobombax septenatum (Jacq.) Dugand & Malvaceae & Ceibo \\
\hline Psidium sartorianum (O. Berg) Nied. & Myrtaceae & Cacique \\
\hline Quercus oleoides Schltdl. y Cham. & Fagaceae & Encino \\
\hline Rehdera trinervis (S.F. Blake) Moldenke & Verbenaceae & Melón/Yayo \\
\hline Roupala montana Aubl. & Proteaceae & Danto \\
\hline Sapium glandulosum (L.) Morong & Euphorbiaceae & Yos \\
\hline Schefflera morototoni (Aubl.) Maguire, Steyerm. & Araliaceae & Pava, probado, fosoforillo \\
\hline Sebastiana pavoniana Mull. Arg. & Euphorbiaceae & Palo de sapo \\
\hline Semialarium mexicanum (Miers) Mennega & Salicaceae & Guácharo \\
\hline Sideroxylon capiri (A. DC.) Pittier & Sapotaceae & Tempisque \\
\hline Simarouba glauca DC. & Simaroubaceae & Aceituno \\
\hline Sloanea terniflora (Moc. y Sesse ex DC.) Sta & Elaeocarpaceae & Pica pica, terciopelo \\
\hline Spondias mombin L. & Anacardiaceae & Jobo \\
\hline Swartzia nicaraguensis (Britton y Rose) Standl. & Fabaceae/Papilionoideae & Carboncillo \\
\hline Swietenia macrophylla King & Meliaceae & Caoba \\
\hline Tabebuia chrysantha (Jacq.) G. Nicholson & Bignoniaceae & Cortez amarillo \\
\hline Tabebuia impetiginosa (Mart. ex DC.) Standl. & Bignoniaceae & Cortez negro \\
\hline Tabebuia ochracea (Cham.) Standl. & Bignoniaceae & Cortez amarillo \\
\hline Wimmeria bartlettii Lundell & Celastraceae & Quebracha \\
\hline Xylopia frutescens Aubl. & Annonaceae & Pavito, malagueto \\
\hline Zuelania guidonia (Sw.) Britton y Millsp. & Salicaceae & Plomo \\
\hline
\end{tabular}

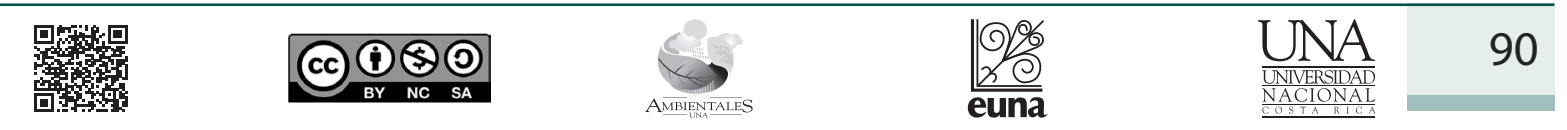




\section{Revista de Sosde 1980 CIENCIAS AMBIENTALES Tropical Journal of Environmental Sciences}

Revista de Ciencias Ambientales (Trop J Environ Sci). EISSN: 2215-3896. (Enero-Junio, 2019) . Vol 53(1): 70-91. Dol: http://dx.doi.org/10.15359/rca.53-1.4 Open Access: www.revistas.una.ac.cr/ambientales EMAlL: revista.ambientales@una.ac.cr

Barquero-Elizondo A. I., Aguilar-Monge I., Méndez-Cartín A.L, Hernández-Sánchez G., Sánchez-Toruño H., Montero-Flores W. Herrera-González E., Sánchez-Chaves L. A., Barrantes-Vásquez A., Gutiérrez-Leitón M., Mesén-Montano I., Bullé-Bueno F.

Apéndice 2. Lista de plantas en flor colectadas en las parcelas y sus alrededores

\begin{tabular}{|c|c|c|}
\hline Nombre científico & Familia & Nombre común \\
\hline \multicolumn{3}{|c|}{ Árboles } \\
\hline Muntingia calabura L. & Muntingiaceae & Capulín \\
\hline \multicolumn{3}{|c|}{ Arbustos } \\
\hline Alibertia edulis (Rich.) A. Rich. ex DC. & Rubiaceae & Trompillo \\
\hline Aphelandra scabra (Vahl) Sm. & Acanthaceae & Cola de gallo \\
\hline Calliandra rubescens (M. Martens y Galeotti) Standl. & Fabaceae/Mimosoideae & Plumerillo \\
\hline Helicteres guazumifolia Kunth & Malvaceae & Rabo de chancho \\
\hline Jacquinia nervosa C. Presl & Primulaceae & Siempreverde \\
\hline Quassia amara L. & Simaroubaceae & Hombre grande \\
\hline Vachellia farnesiana (L.) Wight y Arn. & Fabaceae/Mimosoideae & Cornizuelo \\
\hline Varronia guanacastensis (Standl.) J.S. Mill. & Boraginaceae & Varilla negra \\
\hline Varronia inermis (Mill.) Borhidi & Boraginaceae & Varilla negra \\
\hline \multicolumn{3}{|c|}{ Hierbas } \\
\hline Baltimora recta $\mathrm{L}$. & Asteraceae & ND \\
\hline Calea jamaicensis (L.) L. & Asteraceae & ND \\
\hline Hyptis suaveolens (L.) Poit. & Lamiaceae & ND \\
\hline Kallstroemia pubescens (G. Don) Dandy & Zygophyllaceae & Verdolaga \\
\hline Lantana camara $\mathrm{L}$. & Lamiaceae & Siete negritos \\
\hline Mimosa pigra L. & Fabaceae/Mimosoideae & Dormilona \\
\hline Oplismenus burmannii (Retz.) P. Beauv. & Poaceae & ND \\
\hline Stachytarpheta jamaicensis (L.) Vahl & Lamiaceae & Rabo de zorro \\
\hline Turnera diffusa Willd. & Passifloraceae & ND \\
\hline Turnera ulmifolia L. & Passifloraceae & Damiana \\
\hline Waltheria glomerata C. Presl & Malvaceae & ND \\
\hline Waltheria indica $\mathrm{L}$. & Malvaceae & ND \\
\hline \multicolumn{3}{|c|}{ Lianas herbáceas } \\
\hline Herissantia crispa (L.) Brizicky & Malvaceae & ND \\
\hline Ipomoea sp. & Convolvulaceae & Churristate \\
\hline Merremia cissoides (Lam.) Hallier f. & Convolvulaceae & ND \\
\hline Mimosa albida Humb. y Bonpl. ex Willd. & Fabaceae/Mimosoideae & Rabo de iguana \\
\hline Stigmaphyllon ellipticum (Kunth) A. Juss. & Malpighiaceae & ND \\
\hline \multicolumn{3}{|c|}{ Lianas leñosas } \\
\hline Adenocalymma inundatum Mart. ex DC. & Bignoniaceae & ND \\
\hline Arrabidaea patellifera (Schltdl.) Sandwith & Bignoniaceae & ND \\
\hline Mascagnia sp. & Malpighiaceae & $\mathrm{ND}$ \\
\hline
\end{tabular}

(cc)

